Paulo César Andrade Almeida

Anadergh Barbosa-Branco ${ }^{2}$

\section{Acidentes de trabalho no Brasil: prevalência, duração e despesa previdenciária dos auxílios-doença *}

\author{
Work-related injury and illness in Brazil: prevalence, \\ duration, and incapacity benefit expenditure
}

\footnotetext{
${ }^{1}$ Mestrando em Ciências da Saúde na Faculdade de Ciências da Saúde da Universidade de Brasília (UnB), Brasília, DF, Brasil.

2 Pós-Doutor. Docente do Programa de Pós-Graduação em Ciências da Saúde da Universidade de Brasília (UnB), Brasília, DF, Brasil.

*O presente trabalho não foi subvencionado, não é baseado em tese e não foi apresentado em reunião científica. Este artigo fará parte da dissertação de mestrado de Paulo César Andrade Almeida, a ser submetida ao Programa de Pós-Graduação da Faculdade de Ciências da Saúde da Universidade de Brasília.

\section{Contato:}

Paulo César Andrade Almeida

Ministério da Previdência Social. Esplanada dos Ministérios, Bloco F, Edifício Anexo, Ala B, Sala 434 - Brasília-DF

CEP: 70059-900

E-mail:

pcdfpcdf@gmail.com
}

Recebido: 01/02/2011

Revisado: 27/10/2011

Aprovado: 03/11/2011

\begin{abstract}
Resumo
Objetivo: estimar a prevalência, a duração e a despesa previdenciária dos benefícios de Auxílio-Doença por Acidente do Trabalho (ADAT) pagos pelo Instituto Nacional do Seguro Social (INSS) aos segurados empregados no Brasil em 2008. Método: As variáveis ADAT foram estratificadas segundo sexo, idade e agravos categorizados em capítulos da CID-10. Resultado: foram concedidos 306.908 ADAT com prevalência de 94,2 por 10.000 vínculos. Os agravos mais prevalentes foram os do capítulo XIX - Lesões, envenenamento e algumas outras consequências de causas externas, do XIII - Doenças do sistema osteomuscular e do tecido conjuntivo, e do V - Transtornos mentais e comportamentais. A prevalência dos benefícios foi maior no sexo masculino $(111,2)$ e na faixa etária $\geq 40$ anos $(116,9)$. A duração média foi de $75 \pm 64$ dias e a despesa média de $\mathrm{R} \$ 2.181,00 \pm 2.769,00$, sendo maior para o Capítulo II - Neoplasias (R\$ 5.083,00 \pm 5.702,00). A despesa-dia média foi $\mathrm{R} \$ 29,00 \pm$ 18,00, sendo superior para o Capítulo V (R\$ 39,00 \pm 23,00). A idade $\geq 40$ anos apresentou maior prevalência, duração e despesa entre todos os capítulos, à exceção do XIX, que foi o mais prevalente entre os mais jovens. Conclusão: os dados de ADAT são sugestivos de subcaracterização da relação com o trabalho. A alta prevalência de agravos do Capítulo XIX sugere precariedade das medidas de segurança no trabalho. Homens e mulheres apresentam diferentes perfis de acidentabilidade sem interferência na duração do benefício.
\end{abstract}

Palavras-chave: acidente de trabalho; incapacidade laboral; auxílio-doença; custo; despesa dos benefícios.

\begin{abstract}
Objective: To estimate the prevalence, duration, and expenditure with workrelated incapacity benefits (WRIB) of the Brazilian National Institute of Social Welfare, in 2008. Method: WRIB variables were stratified according to gender, age, and diseases categorized into ICD-10 chapters. Results: There were 306,908 WRIB granted in 2008, with the prevalence of 94.2 per 10,000 insured workers. The most prevalent ICD-10 chapters were: XIX - Injury, poisoning and some other external cause consequences, XIII - Diseases of the musculoskeletal system and connective tissue, and V-Mental and behavioral disorders. The prevalence was higher among men (111.2) and those $\geq 40$ years of age (116.9). Benefit was received for $75 \pm$ 64 days independently of gender, and the average expenditure was $R \$ 2,181.00 \pm$ 2,769.00, being higher for Chapter II - Neoplasms (R\$ 5,083.00 \pm 5,702.00). The average daily expenditure was $R \$ 29.00 \pm 18.00$, being higher for Chapter $V(R \$$ $39.00 \pm 23.00$ ). Those $\geq 40$ years of age were the most prevalent, received benefits for the longest period and the highest expenses in all chapters, except in XIX, in which the highest prevalence was of younger workers. Conclusion: WRIB data suggested underreporting of the work-relatedness of diseases. The prevalence of chapter XIX suggested that work environment safety measures were inadequate. Men and women showed different profiles of work disability; however, there was no gender difference in the length of benefit.
\end{abstract}

Keywords: occupational injury and illness; work disability; incapacity benefit; cost; benefit expenditure. 


\section{Introdução}

Os agravos à saúde decorrentes de acidentes de trabalho (AT) podem ou não resultar em incapacidade laboral, quer seja temporária ou permanente (BRASIL, 2009a).

Apesar dos ATs representarem importante problema social, econômico e de saúde pública, os pesquisadores têm sido quase unânimes em afirmar a subnotificação desses eventos, subdimensionando o problema (BENAVIDES et al., 2003; ROSENMAN et al., 2006; SALINAS-TOVAR et al., 2004). A Organização Internacional do Trabalho (OIT) estima que apenas 3,9\% dos ATs são notificados (HÄMÄLÄINEN; TAKALA; SAARELA, 2006). Países desenvolvidos, como a maioria dos europeus, EUA, Canadá, Japão, Austrália e Nova Zelândia, notificam em torno de $62,0 \%$ dos ATs, enquanto os da América Latina e do Caribe, em torno de 7,6\%; nos países da África Subsaariana e do Oriente Médio, na Índia e na China, a notificação dos ATs está abaixo de 1,0\%.

$\mathrm{O}$ conceito de AT e as proteções sociais que destes decorrem variam consideravelmente, o que dificulta a comparação dos dados entre países (EUROPE, 2004) ou mesmo entre estados de um mesmo país, como é o caso dos EUA (FULTON-KEHOE et al., 2000; LEIGH; McCURDY, 2006). Na legislação previdenciária brasileira, os acidentes de trabalho compreendem as lesões, as doenças profissionais, as doenças do trabalho e os acidentes de trajeto, além de outras situações previstas na legislação (BRASIL, 1991). No Brasil, principalmente até abril de 2007, os registros de AT têm sido reconhecidos como altamente subnotificados (BINDER; CORDEIRO, 1997; SANTANA; NOBRE; WALDVOGEL, 2005). No período antecedente a abril de 2007, no Regime Geral de Previdência Social (RGPS), maior regime de previdência brasileiro, administrado pelo Instituto Nacional do Seguro Social (INSS), a notificação de um AT dependia, quase que exclusivamente, da emissão da Comunicação de Acidente do Trabalho (CAT), sob a responsabilidade primária do empregador. Em abril de 2007, após inúmeras evidências científicas dessa subnotificação (BARBOSA-BRANCO; ROMARIZ, 2006; FACCHINI et al., 2005; SANTANA, 2005), o INSS implementou o Nexo Técnico Epidemiológico Previdenciário - NTEP (BRASIL, 2007).

O NTEP decorre de um estudo de coorte, de base populacional, para estabelecimento de correlações entre grupos de diagnósticos da incapacidade para o trabalho e atividades econômicas às quais os trabalhadores incapacitados estão vinculados (OLIVEIRA-ALBUQUERQUE; BARBOSA-BRANCO, 2008). O NTEP disponibiliza à Perícia Médica do INSS um perfil epidemiológico de adoecimento e de aciden- tabilidade dos empregados de todas as atividades econômicas do país. Essa metodologia de caracterização da natureza acidentária oferece ao processo de concessão de benefício uma nova opção para estabelecimento de nexo técnico entre o trabalho/atividade econômica e o agravo, independente, mas não excludentemente, da emissão da CAT.

Apesar dos avanços ocorridos a partir de abril 2007, mesmo sem a dependência exclusiva da CAT para notificação de AT, a subnotificação ainda persiste, principalmente em relação aos acidentes com duração do absenteísmo inferior a dezesseis dias consecutivos. Para esses afastamentos, não há concessão de benefício e consequentemente não há aplicação do NTEP.

Mesmo considerado esse contexto de subnotificação, os gastos decorrentes dos ATs são expressivos. Em 2003, a OIT estimou esses gastos em torno de 4\% do Produto Interno Bruto - PIB (INTERNATIONAL LABOUR ORGANIZATION, 2003), variando entre os países conforme os específicos graus de desenvolvimento. Em 2001, a European Agency for Safety and Health at Work (Osha-EU) estimou que os gastos dos ATs nos países membros da Comunidade Econômica Europeia variaram de 2,6\% a 3,8\% do PIB (RIKHARDSSON, 2004).

Os gastos com esse tipo de agravo geralmente são classificados em diretos e indiretos (FREEMANA et al., 2001; LUNES, 1997). Os gastos diretos podem ser considerados aqueles nos quais a relação de consequência direta com o acidente é de fácil percepção, como assistência à saúde, pagamento de benefícios previdenciários (auxílio-doença, aposentadoria por invalidez e pensão por morte), entre outros.

Classificam-se como gastos indiretos os relacionados às perdas salariais dos trabalhadores, quando não totalmente compensadas com o recebimento dos benefícios previdenciários, os salários do trabalhador afastado (primeiros 15 dias) e do trabalhador substituto, o treinamento do trabalhador substituto, os encargos trabalhistas (Fundo de Garantia por Tempo de Serviço - FGTS), tributários e advocatícios, bem como os danos patrimoniais, entre outros. Esses gastos são de percepção mais moderada.

Apesar dos autores apresentarem distintas razões entre os gastos diretos e os indiretos (LARSSON; BETTS, 1996), a ilustração dessa relação é comumente estabelecida por meio de uma analogia com a figura de um iceberg, na qual os gastos diretos são representados pela parte visível e os indiretos, pela parte submersa. Além dos gastos diretos e indiretos, há outros de difícil percepção que estão relacionados à perda da qualidade de vida (MORAES et al., 2006). 
Este estudo restringe-se aos gastos dos benefícios Auxílio-Doença por Acidente do Trabalho (ADAT), pagos aos empregados segurados do RGPS ${ }^{3}$ a título de reposição salarial, a partir do $16^{\circ}$ dia de afastamento por incapacidade temporária para o trabalho (BRASIL, 1991).

Ressalta-se que o gênero gastos compreende as espécies custos, perdas e despesas e que, no âmbito da administração pública, os gastos com os pagamentos de benefícios são considerados despesas públicas, uma vez que se inserem no contexto da contabilidade pública (BRASIL, 1964).

Considerando a carência de pesquisas no Brasil acerca das repercussões econômicas dos ATs, o presente estudo objetiva estimar a prevalência, a duração e a despesa previdenciária dos ADAT e a influência de fatores como o grupo de agravos, de acordo com a Classificação Estatística Internacional de Doenças e Problemas Relacionados à Saúde $-10^{\mathrm{a}}$ Revisão - CID-10 (BRASIL, 2011), o sexo e a idade do empregado nesses indicadores.

\section{Método}

\section{População de estudo}

No Brasil, em 2008, a População Ocupada (INSTITUTO BRASILEIRO DE GEOGRAFIA E ESTATÍSTICA, 2010), suscetível à incapacidade laboral, foi estimada em 92.394 .585 pessoas, das quais 40.425 .749 $(43,8 \%)$ são contribuintes do RGPS (BRASIL, 2010). Dentre os segurados do RGPS, definiu-se como população de estudo o número médio mensal de vínculos (NMV) dos empregados naquele ano, que totalizou 32.590.239. O NMV corresponde à soma dos meses trabalhados para cada vínculo dividido por 12 (BRASIL, 2009b).

\section{Definição de caso}

Foi considerado caso todo benefício concedido no período de 01/01/2008 a 31/12/2008 a título de reposição salarial quando da incapacidade temporária superior a quinze dias consecutivos decorrente de qualquer situação que se enquadre na definição e na equiparação legal de acidente de trabalho (BRASIL, 1991). Essa reposição salarial é denominada Auxílio-Doença por Acidente do Trabalho (ADAT).

\section{Fonte de dados}

Os dados analisados nesta pesquisa foram extraídos do Sistema Único de Benefícios (SUB) e do Cadastro Nacional de Informações Socais (CNIS), ambos gerenciados pela Empresa de Tecnologia e Informações da Previdência Social (Dataprev), empresa pública vinculada ao Ministério da Previdência Social (MPS).

O SUB é um sistema de grande porte que unifica todos os registros de concessão e manutenção de benefícios administrados pelo INSS.

O CNIS é um sistema alimentado e atualizado, entre outras fontes, por intermédio da Guia de Recolhimento do Fundo de Garantia por Tempo de Serviço e de informações à Previdência Social (GFIP), as quais são declaradas mensalmente, de forma compulsória, pelas empresas.

\section{Tratamento dos dados}

Após a extração, no SUB, das informações dos ADAT referentes à duração, à despesa, ao código do capítulo da CID-10, ao sexo e à idade do empregado beneficiário e, no CNIS, das informações referentes ao NMV, ao sexo e à idade de todos os empregados, os dados foram armazenados no sistema de gerenciamento de banco de dados Microsoft Office Access ${ }^{\circledR}$. Os ADAT que tiveram as informações acima elencadas como ausentes ou incompletas foram excluídos da análise. Para a análise dos capítulos da CID-10, foram agrupados em "Outros" os capítulos III - Doenças do sangue e dos órgãos hematopoéticos e alguns transtornos imunitários, IV - Doenças endócrinas, nutricionais e metabólicas, XV - Gravidez, parto e puerpério e XVII - Algumas afecções originadas no período perinatal, por apresentarem, em conjunto, tão somente 501 ADAT, sendo que, entre esses, os capítulos XV e XVII referem-se a concessões restritas ao sexo feminino.

\section{Análise dos dados}

Por meio do programa de planilha eletrônica Microsoft Office Excel ${ }^{\circledR}$, foram realizados cálculos de prevalência, ${ }^{4}$ duração média, despesa média e despesa-dia dos ADAT, segundo os agravos agrupados pelo capítulo CID-10, o sexo (masculino ou feminino) e a faixa etária $(<40$ ou $\geq 40$ anos). A prevalência é apresentada como o número de casos de ADAT por 10.000 vínculos. A duração média é obtida por meio

\footnotetext{
${ }^{3}$ Classificam-se como segurados do RGPS (Regime Geral da Previdencia Social): empregados, trabalhadores avulsos, empregados domésticos, contribuintes individuais, segurados especiais e segurados facultativos (BRASIL, 1991).

${ }^{4}$ Em que pese os ADAT serem referentes aos benefícios concedidos no ano de 2008, o que em princípio daria ensejo a cálculo de incidência, a presente pesquisa faz um corte seccional no ano de 2008, sem avaliação das entradas e saídas dos trabalhadores e impossibilidade de acompanhamento pessoa-tempo no período analisado.
} 
da divisão do somatório da duração pelo número de casos. A despesa média resulta do somatório da despesa dividida pelo número de casos. A despesa-dia é o resultado da divisão do somatório da despesa pelo somatório da duração. A opção pelo uso complementar da mediana foi feita buscando mostrar possíveis distorções da média como medida de tendência central, trazendo uma visão mais ampla da distribuição dessas variáveis na população de estudo.

Por apresentarem inexpressiva casuística e como para alguns capítulos há restrição de registro ao sexo feminino, todos os resultados dos capítulos CID-10 agrupados em “Outros” não são considerados na análise.

O término da duração dos benefícios foi computado até a data limite de 31/12/2009, que por definição de projeto corresponde à data de extração dos dados.

\section{Resultados}

Em 2008, O INSS concedeu 356.336 ADAT, dos quais $306.908(86,1 \%)$ continham as informações mínimas necessárias para a análise. Esse quantitativo de ADAT resultou em uma prevalência estimada de 94,2 por 10.000 vínculos.

A Tabela 1 apresenta a prevalência de ADAT segundo os agravos do capítulo CID-10, o sexo e a faixa etária do segurado. Os capítulos CID-10 com as maiores prevalências são os capítulos XIX - Lesões, envenenamentos e algumas outras consequências de causas externas (lesões), XIII - Doenças do sistema osteomuscular e tecido conjuntivo (doenças osteomusculares) e V - Transtornos mentais e comportamentais (doenças mentais). Esses três capítulos respondem por 92,5\% da casuística (dado não apresentado).

Tabela 1 Prevalência* de auxílios-doença por acidente do trabalho (ADAT) segundo grupo de agravos por capítulos da CID-10, sexo e faixa etária dos segurados empregados do Regime Geral da Previdência Social, Brasil, 2008

\begin{tabular}{|c|c|c|c|c|c|}
\hline \multirow{2}{*}{$\begin{array}{l}\text { Capítulo } \\
\text { CID-10** }\end{array}$} & \multicolumn{2}{|c|}{ Sexo } & \multicolumn{2}{|c|}{ Faixa etária } & \multirow{2}{*}{ Total } \\
\hline & Masculino & Feminino & $<40$ anos & $\geq 40$ anos & \\
\hline I & 0,6 & 0,5 & 0,5 & 0,7 & 0,5 \\
\hline II & 0,1 & 0,1 & 0,1 & 0,3 & 0,1 \\
\hline V & 2,9 & 5,5 & 3,3 & 4,7 & 3,7 \\
\hline VI & 1,0 & 5,1 & 1,7 & 4,3 & 2,5 \\
\hline VII & 0,6 & 0,2 & 0,4 & 0,5 & 0,4 \\
\hline VIII & 0,1 & 0,1 & 0,1 & 0,2 & 0,1 \\
\hline IX & 1,1 & 1,3 & 0,5 & 2,5 & 1,1 \\
\hline$x$ & 0,4 & 0,8 & 0,4 & 0,9 & 0,5 \\
\hline XI & 1,5 & 0,2 & 0,8 & 1,4 & 1,0 \\
\hline XII & 0,4 & 0,3 & 0,3 & 0,5 & 0,3 \\
\hline XIII & 28,2 & 37,3 & 22,4 & 49,4 & 30,5 \\
\hline XIV & 0,1 & 0,0 & 0,1 & 0,1 & 0,1 \\
\hline XIX & 73,8 & 24,8 & 54,1 & 51,0 & 53,1 \\
\hline XXI & 0,2 & 0,2 & 0,2 & 0,3 & 0,2 \\
\hline Outros & 0,1 & 0,2 & 0,1 & 0,2 & 0,2 \\
\hline Total & 111,2 & 76,5 & 84,8 & 116,9 & 94,2 \\
\hline
\end{tabular}

" Prevalência por 10.000 vínculos.

***: Capítulos CID-10 - Descrição: I - Algumas doenças infecciosas e parasitárias; II - Neoplasias (tumores); V - Transtornos mentais e comportamentais; VI - Doenças do sistema nervoso; VII Doenças do olho e anexos; VIII - Doenças do ouvido e da apófise mastoide; IX - Doenças do aparelho circulatório; X - Doenças do aparelho respiratório; XI - Doenças do aparelho digestivo; XII - Doenças da pele e do tecido subcutâneo; XIII - Doenças sistema osteomuscular e tecido conjuntivo; XIV Doenças do aparelho geniturinário; XIX - Lesões envenenamento e algumas outras consequências causas externas; XXI - Contatos com serviços de saúde. 
A prevalência estimada de ADAT é $45,4 \%$ maior entre os homens $(111,2 / 10.000)$ comparada àquela entre as mulheres (76,5/10.000), sendo o Capítulo XI - Doenças do aparelho digestivo o que apresenta a maior razão de prevalências entre os sexos $(7,5)$. As mulheres apresentam maior prevalência das doenças mentais (V), das doenças do sistema nervoso (VI), do aparelho circulatório (IX), do aparelho respiratório (X) e do sistema osteomuscular (XIII). A prevalência estimada na faixa etária $\geq 40$ anos $(116,9)$ supera em $37,8 \%$ a faixa $<40$ anos $(84,8)$. Vale ressaltar que apenas o capítulo da CID-10 das lesões (XIX) apresenta maior prevalência na menor faixa etária $(<40$ anos).

A Tabela 2 apresenta a duração média dos ADAT para 2008 segundo o capítulo da CID-10, o sexo e a faixa etária do empregado. A duração média dos benefícios (75 \pm 64 dias; mediana $=59$ dias) não apresenta diferença entre os sexos. Os ADAT referentes às neoplasias (II) apresentam a maior duração média (146 \pm 106 dias; mediana $=121$ dias $)$, contrapondo-se aos das doenças do aparelho digestivo (54 \pm 33 dias; mediana $=47$ dias). À exceção das neoplasias (Capítulo - II), a duração média dos ADAT é maior na faixa etária $\geq 40$ anos.

Dos 306.908 ADAT, apenas 13 benefícios tiveram mais de dois anos de duração, não trazendo prejuízos à comparação com dados de outros estudos cujo acompanhamento da duração tenha sido realizado por período superior.

Tabela 2 Duração média dos auxílios-doença por acidente do trabalho (ADAT) segundo grupo de agravos por capítulo CID-10, sexo e faixa etária dos segurados empregados do Regime Geral da Previdência Social, Brasil, 2008

\begin{tabular}{|c|c|c|c|c|c|c|c|c|c|c|c|c|c|c|c|}
\hline \multirow[t]{2}{*}{$\begin{array}{l}\text { Capítulo } \\
\text { CID-10 }\end{array}$} & \multicolumn{6}{|c|}{ Sexo } & \multicolumn{6}{|c|}{ Faixa etária } & \multicolumn{3}{|c|}{ Total } \\
\hline & $\mathrm{M}^{*}$ & $\begin{array}{l} \pm \mathrm{DP} \\
\text { (dias) }\end{array}$ & MED & M & $\begin{array}{l} \pm \mathrm{DP} \\
\text { (dias) }\end{array}$ & MED & M & $\begin{array}{l} \pm \mathrm{DP} \\
\text { (dias) }\end{array}$ & MED & $M$ & $\begin{array}{l} \pm \mathrm{DP} \\
\text { (dias) }\end{array}$ & MED & M & $\begin{array}{l} \pm \mathrm{DP} \\
\text { (dias) }\end{array}$ & MED \\
\hline I & 105 & 77 & 87 & 97 & 71 & 81 & 96 & 73 & 77 & 112 & 78 & 93 & 102 & 75 & 85 \\
\hline II & 151 & 109 & 130 & 134 & 100 & 114 & 149 & 109 & 115 & 145 & 106 & 122 & 146 & 106 & 121 \\
\hline V & 86 & 72 & 69 & 75 & 61 & 55 & 76 & 62 & 63 & 86 & 72 & 69 & 80 & 66 & 65 \\
\hline VI & 94 & 77 & 75 & 89 & 72 & 73 & 89 & 73 & 72 & 91 & 73 & 75 & 90 & 73 & 74 \\
\hline VII & 85 & 87 & 60 & 60 & 75 & 41 & 72 & 75 & 53 & 97 & 99 & 62 & 81 & 85 & 56 \\
\hline VIII & 99 & 99 & 65 & 113 & 103 & 81 & 92 & 97 & 55 & 111 & 102 & 78 & 102 & 100 & 67 \\
\hline IX & 89 & 77 & 69 & 60 & 57 & 46 & 61 & 59 & 46 & 85 & 74 & 64 & 77 & 70 & 56 \\
\hline$x$ & 101 & 99 & 66 & 87 & 85 & 64 & 89 & 90 & 64 & 98 & 93 & 66 & 93 & 92 & 65 \\
\hline XI & 55 & 31 & 47 & 49 & 49 & 45 & 53 & 32 & 46 & 56 & 33 & 48 & 54 & 33 & 47 \\
\hline XII & 75 & 78 & 52 & 79 & 86 & 48 & 72 & 78 & 47 & 82 & 85 & 54 & 76 & 81 & 50 \\
\hline XIII & 81 & 70 & 63 & 79 & 70 & 61 & 73 & 65 & 58 & 86 & 74 & 68 & 80 & 70 & 62 \\
\hline XIV & 121 & 106 & 86 & 145 & 118 & 107 & 105 & 96 & 75 & 149 & 117 & 115 & 125 & 108 & 88 \\
\hline XIX & 71 & 58 & 56 & 65 & 53 & 51 & 68 & 55 & 54 & 74 & 61 & 60 & 70 & 57 & 55 \\
\hline XXI & 63 & 76 & 61 & 101 & 74 & 63 & 74 & 65 & 55 & 97 & 85 & 72 & 84 & 75 & 62 \\
\hline Outros & 207 & 138 & 127 & 152 & 143 & 107 & 154 & 138 & 96 & 195 & 139 & 180 & 174 & 140 & 117 \\
\hline Total & 75 & 63 & 59 & 75 & 65 & 59 & 70 & 60 & 56 & 82 & 70 & 64 & 75 & 64 & 59 \\
\hline
\end{tabular}

* $\mathrm{M}=$ Duração média.

$\pm \mathrm{DP}=$ Desvio-padrão da duração.

MED = Mediana da duração.

**: Capítulos CID-10 - Descrição: I - Algumas doenças infecciosas e parasitárias; II - Neoplasias (tumores); V - Transtornos mentais e comportamentais; VI - Doenças do sistema nervoso; VII - Doenças do olho e anexos; VIII - Doenças do ouvido e da apófise mastoide; IX - Doenças do aparelho circulatório; X - Doenças do aparelho respiratório; XI - Doenças do aparelho digestivo; XII - Doenças da pele e do tecido subcutâneo; XIII - Doenças sistema osteomuscular e tecido conjuntivo; XIV - Doenças do aparelho geniturinário; XIX - Lesões envenenamento e algumas outras consequências causas externas; XXI - Contatos com serviços de saúde. 
A Tabela 3 apresenta os valores da despesa média dos ADAT em 2008 segundo o capítulo da CID10 e o sexo do empregado. A despesa média geral dos ADAT é R\$2.181,00 \pm 2.769,00; mediana $=\mathrm{R} \$$ $1.360,00$. Quando esse indicador é analisado por capítulo da CID-10, as neoplasias (II) registram a maior despesa média $(\mathrm{R} \$ 5.083,00 \pm 5.702,00$; mediana $=$ $\mathrm{R} \$ 3.080,00)$, enquanto as doenças do aparelho digestivo (XI) apresentam a menor despesa média $(\mathrm{R} \$ 1.477,00 \pm 1.179,00$; mediana $=\mathrm{R} \$ 1.135,00)$. A despesa média entre os homens ( $\mathrm{R} \$ 2.273,00 \pm$ $2.802,00$; mediana $=\mathrm{R} \$ 1.439,00)$ é $15,7 \%$ maior que entre as mulheres $(\mathrm{R} \$ 1.964,00 \pm 2.679,00$; mediana $=\mathrm{R} \$ 1.202,00)$ na totalidade dos capítulos analisados. A superioridade das despesas médias entre os homens mantém-se em cada capítulo da CID10 , sendo a maior diferença $(81,3 \%)$ entre os sexos, observada nas doenças do olho (VII).

A Tabela 4 apresenta os valores de despesa média dos ADAT segundo o capítulo CID-10 e a faixa etária do empregado. A diferença da despesa média entre as faixas etárias é de 63,0\%, sendo maior entre os trabalhadores com idade $\geq 40$ anos em todos os capítulos CID-10.

A Tabela 5 apresenta os valores da despesa-dia dos ADAT segundo o capítulo CID-10, o sexo e a faixa etária do empregado. A despesa-dia ( $R \$$ $39,00 \pm 23,00$; mediana $=R \$ 30,00)$ é maior entre as doenças mentais (II) e menor ( $R \$ 24,00 \pm 13,00$; mediana $=R \$ 19,00$ ) entre as doenças da pele. Em relação à despesa-dia dos ADAT segundo o sexo, esse indicador foi maior entre os homens (R\$ $30,00 \pm 18,00$; mediana $=R \$ 23,00$ ), superando as mulheres $(R \$ 26,00 \pm 17,00$; mediana $=R \$ 19,00)$ em 15,4\%. Considerando a idade, os empregados na faixa etária $\geq 40$ anos ( $R \$ 35,00 \pm 21,00$; mediana $=\mathrm{R} \$ 27,00$ ) apresentam despesa-dia 40,0\% maior do que os mais jovens $(\mathrm{R} \$ 25,00 \pm 13,00$; mediana $=\mathrm{R} \$ 20,00$ ).

Tabela 3 Despesa média de auxílios-doença por acidente do trabalho (ADAT) segundo grupo de agravos por capítulo CID-10 e sexo dos segurados empregados do Regime Geral da Previdência Social, Brasil, 2008

\begin{tabular}{|c|c|c|c|c|c|c|c|c|c|}
\hline \multirow{2}{*}{$\begin{array}{l}\text { Capítulo } \\
\text { CID-10*** }\end{array}$} & \multicolumn{3}{|c|}{ Masculino } & \multicolumn{3}{|c|}{ Feminino } & \multicolumn{3}{|c|}{ Total } \\
\hline & $M^{*}$ & $\begin{array}{c} \pm D P \\
\text { (Reais) }\end{array}$ & MED & $M$ & $\begin{array}{c} \pm D P \\
\text { (Reais) }\end{array}$ & MED & $M$ & $\begin{array}{c} \pm D P \\
\text { (Reais) }\end{array}$ & $M E D$ \\
\hline I & 2.901 & 3.155 & 2.001 & 2.607 & 2.678 & 1.737 & 2.806 & 3.012 & 1.932 \\
\hline II & 5.533 & 6.095 & 3.507 & 3.971 & 4.426 & 2.510 & 5.083 & 5.702 & 3.080 \\
\hline V & 3.717 & 4.130 & 2.477 & 2.578 & 3.246 & 1.593 & 3.106 & 3.725 & 1.948 \\
\hline $\mathrm{VI}$ & 3.638 & 4.192 & 2.335 & 2.517 & 3.242 & 1.532 & 2.796 & 3.535 & 1.673 \\
\hline VII & 2.357 & 3.060 & 1.383 & 1.300 & 1.640 & 870 & 2.195 & 2.913 & 1.269 \\
\hline VIII & 3.745 & 4.949 & 1.868 & 2.736 & 3.293 & 1.425 & 3.522 & 4.648 & 1.736 \\
\hline IX & 3.176 & 3.695 & 2.027 & 1.384 & 1.629 & 964 & 2.428 & 3.137 & 1.418 \\
\hline$x$ & 3.172 & 4.281 & 1.672 & 2.059 & 2.628 & 1.282 & 2.565 & 3.521 & 1.411 \\
\hline$X I$ & 1.514 & 1.173 & 1.181 & 1.063 & 1.164 & 809 & 1.477 & 1.179 & 1.135 \\
\hline XII & 1.935 & 2.661 & 1.108 & 1.577 & 2.081 & 861 & 1.820 & 2.494 & 1.047 \\
\hline XIII & 2.897 & 3.598 & 1.757 & 2.089 & 2.930 & 1.243 & 2.534 & 3.339 & 1.480 \\
\hline XIV & 3.453 & 4.018 & 1.882 & 3.446 & 3.603 & 1.919 & 3.452 & 3.938 & 1.882 \\
\hline$X I X$ & 1.932 & 2.170 & 1.310 & 1.523 & 1.784 & 1.051 & 1.862 & 2.115 & 1.259 \\
\hline XXI & 2.737 & 3.731 & 1.574 & 2.387 & 3.526 & 1.311 & 2.595 & 3.650 & 1.416 \\
\hline Outros & 6.191 & 7.058 & 2.289 & 4.846 & 6.199 & 1.331 & 5.579 & 6.708 & 1.743 \\
\hline Total & 2.273 & 2.802 & 1.439 & 1.964 & 2.679 & 1.202 & 2.181 & 2.769 & 1.360 \\
\hline
\end{tabular}

* $\mathrm{M}=$ Despesa média.

$\pm \mathrm{DP}=$ Desvio-padrão da despesa.

MED = Mediana da despesa.

*** Capítulos CID-10 - Descrição: I - Algumas doenças infecciosas e parasitárias; II - Neoplasias (tumores); V - Transtornos mentais e comportamentais; VI - Doenças do sistema nervoso; VII - Doenças do olho e anexos; VIII - Doenças do ouvido e da apófise mastoide; IX - Doenças do aparelho circulatório; X - Doenças do aparelho respiratório; XI - Doenças do aparelho digestivo; XII - Doenças da pele e do tecido subcutâneo; XIII - Doenças sistema osteomuscular e tecido conjuntivo; XIV - Doenças do aparelho geniturinário; XIX - Lesões envenenamento e algumas outras consequências causas externas; XXI - Contatos com serviços de saúde. 
Tabela 4 Despesa média de auxílios-doença por acidente do trabalho (ADAT) segundo grupo de agravos por capítulo CID-10 e faixa etária dos segurados empregados do Regime Geral da Previdência Social, Brasil, 2008

\begin{tabular}{|c|c|c|c|c|c|c|c|c|c|}
\hline \multirow{2}{*}{$\begin{array}{l}\text { Capítulo } \\
\text { CID-10*** }\end{array}$} & \multicolumn{3}{|c|}{$<40$ anos } & \multicolumn{3}{|c|}{$\geq 40$ anos } & \multicolumn{3}{|c|}{ Total } \\
\hline & $M^{*}$ & $\begin{array}{c} \pm D P \\
\text { (Reais) }\end{array}$ & MED & $M$ & $\begin{array}{c} \pm D P \\
\text { (Reais) }\end{array}$ & MED & $M$ & $\begin{array}{c} \pm D P \\
\text { (Reais) }\end{array}$ & MED \\
\hline I & 2.296 & 2.419 & 1.611 & 3.563 & 3.592 & 2.531 & 2.806 & 3.012 & 1.932 \\
\hline II & 4.078 & 3.997 & 2.510 & 5.534 & 6.275 & 3.463 & 5.083 & 5.702 & 3.080 \\
\hline V & 2.549 & 3.041 & 1.657 & 4.018 & 4.486 & 2.712 & 3.106 & 3.725 & 1.948 \\
\hline VI & 2.295 & 2.598 & 1.499 & 3.251 & 4.157 & 1.881 & 2.796 & 3.535 & 1.673 \\
\hline VII & 1.648 & 1.920 & 1.119 & 3.207 & 3.975 & 1.801 & 2.195 & 2.913 & 1.269 \\
\hline VIII & 2.428 & 3.130 & 1.344 & 4.516 & 5.512 & 2.263 & 3.522 & 4.648 & 1.736 \\
\hline IX & 1.533 & 1.840 & 1.045 & 2.878 & 3.533 & 1.700 & 2.428 & 3.137 & 1.418 \\
\hline$x$ & 2.231 & 3.162 & 1.282 & 2.930 & 3.845 & 1.636 & 2.565 & 3.521 & 1.411 \\
\hline$X I$ & 1.243 & 936 & 1.011 & 1.785 & 1.379 & 1.391 & 1.477 & 1.179 & 1.135 \\
\hline XII & 1.534 & 1.923 & 917 & 2.188 & 3.039 & 1.254 & 1.820 & 2.494 & 1.047 \\
\hline XIII & 1.982 & 2.539 & 1.245 & 3.108 & 3.923 & 1.820 & 2.534 & 3.339 & 1.480 \\
\hline XIV & 2.422 & 2.997 & 1.429 & 4.678 & 4.544 & 2.958 & 3.452 & 3.938 & 1.882 \\
\hline XIX & 1.603 & 1.719 & 1.134 & 2.493 & 2.757 & 1.670 & 1.862 & 2.115 & 1.259 \\
\hline XXI & 2.017 & 2.780 & 1.170 & 3.348 & 4.434 & 1.895 & 2.595 & 3.650 & 1.416 \\
\hline Outros & 4.254 & 6.031 & 2.268 & 6.971 & 7.111 & 4.881 & 5.579 & 6.708 & 1.743 \\
\hline Total & 1.764 & 2.094 & 1.185 & 2.876 & 3.517 & 1.674 & 2.181 & 2.769 & 1.360 \\
\hline
\end{tabular}

* $\mathrm{M}=$ Despesa média.

$\pm \mathrm{DP}=$ Desvio-padrão da despesa.

MED = Mediana da despesa.

*** Capítulos CID-10 - Descrição: I - Algumas doenças infecciosas e parasitárias; II - Neoplasias (tumores); V - Transtornos mentais e comportamentais; VI - Doenças do sistema nervoso; VII - Doenças do olho e anexos; VIII - Doenças do ouvido e da apófise mastoide; IX - Doenças do aparelho circulatório; X - Doenças do aparelho respiratório; XI - Doenças do aparelho digestivo; XII - Doenças da pele e do tecido subcutâneo; XIII - Doenças sistema osteomuscular e tecido conjuntivo; XIV - Doenças do aparelho geniturinário; XIX - Lesões envenenamento e algumas outras consequências causas externas; XXI - Contatos com serviços de saúde.

Tabela 5 Despesa-dia de auxílios-doença por acidente do trabalho (ADAT) segundo grupo de agravos por capítulo CID-10, sexo e faixa etária dos segurados empregados do Regime Geral da Previdência Social, Brasil, 2008

\begin{tabular}{|c|c|c|c|c|c|c|c|c|c|c|c|c|c|c|c|}
\hline \multirow{2}{*}{$\begin{array}{l}\text { Capítulo } \\
\text { CID-10*** }\end{array}$} & \multicolumn{3}{|c|}{ Masculino } & \multicolumn{3}{|c|}{ Feminino } & \multicolumn{3}{|c|}{$<40$ anos } & \multicolumn{3}{|c|}{$\geq 40$ anos } & \multicolumn{3}{|c|}{ Total } \\
\hline & $D^{*}$ & $\begin{array}{c} \pm D P \\
\text { (Reais) }\end{array}$ & MED & $D$ & $\begin{array}{c} \pm D P \\
\text { (Reais) }\end{array}$ & MED & $D$ & $\begin{array}{c} \pm D P \\
\text { (Reais) }\end{array}$ & MED & $D$ & $\begin{array}{c} \pm D P \\
\text { (Reais) }\end{array}$ & MED & $D$ & $\begin{array}{c} \pm D P \\
\text { (Reais) }\end{array}$ & MED \\
\hline I & 28 & 17 & 22 & 27 & 19 & 20 & 24 & 15 & 19 & 32 & 20 & 25 & 27 & 18 & 21 \\
\hline II & 37 & 22 & 29 & 30 & 17 & 22 & 27 & 15 & 22 & 38 & 23 & 30 & 35 & 21 & 27 \\
\hline V & 43 & 23 & 36 & 34 & 22 & 24 & 33 & 19 & 26 & 47 & 25 & 40 & 39 & 23 & 30 \\
\hline VI & 39 & 24 & 29 & 28 & 19 & 20 & 26 & 15 & 20 & 36 & 24 & 24 & 31 & 21 & 22 \\
\hline VII & 28 & 16 & 23 & 22 & 16 & 18 & 23 & 12 & 20 & 33 & 20 & 27 & 27 & 16 & 22 \\
\hline VIII & 38 & 20 & 29 & 24 & 18 & 19 & 26 & 14 & 21 & 41 & 22 & 34 & 34 & 20 & 25 \\
\hline IX & 36 & 20 & 30 & 23 & 14 & 19 & 25 & 14 & 21 & 34 & 20 & 26 & 32 & 19 & 24 \\
\hline$x$ & 32 & 17 & 25 & 24 & 14 & 19 & 25 & 13 & 20 & 30 & 18 & 24 & 28 & 16 & 21 \\
\hline$X I$ & 28 & 16 & 23 & 22 & 13 & 17 & 24 & 12 & 21 & 32 & 18 & 27 & 27 & 16 & 23 \\
\hline XII & 26 & 14 & 21 & 20 & 9 & 16 & 21 & 10 & 18 & 27 & 15 & 22 & 24 & 13 & 19 \\
\hline
\end{tabular}

(continua) 
Tabela 5 Despesa-dia de auxílios-doença por acidente do trabalho (ADAT) segundo grupo de agravos por capítulo CID-10, sexo e faixa etária dos segurados empregados do Regime Geral da Previdência Social, Brasil, 2008

(continuação)

\begin{tabular}{|c|c|c|c|c|c|c|c|c|c|c|c|c|c|c|c|}
\hline \multirow{2}{*}{$\begin{array}{l}\text { Capítulo } \\
\text { CID-10** }\end{array}$} & \multicolumn{3}{|c|}{ Masculino } & \multicolumn{3}{|c|}{ Feminino } & \multicolumn{3}{|c|}{$<40$ anos } & \multicolumn{3}{|c|}{$\geq 40$ anos } & \multicolumn{3}{|c|}{ Total } \\
\hline & $D^{*}$ & $\begin{array}{c} \pm D P \\
\text { (Reais) }\end{array}$ & MED & $D$ & $\begin{array}{c} \pm D P \\
\text { (Reais) }\end{array}$ & MED & $D$ & $\begin{array}{c} \pm D P \\
\text { (Reais) }\end{array}$ & MED & $D$ & $\begin{array}{c} \pm D P \\
\text { (Reais) }\end{array}$ & MED & $D$ & $\begin{array}{c} \pm D P \\
\text { (Reais) }\end{array}$ & MED \\
\hline XIII & 36 & 21 & 27 & 27 & 17 & 19 & 27 & 15 & 21 & 36 & 22 & 26 & 32 & 20 & 23 \\
\hline XIV & 29 & 19 & 21 & 24 & 14 & 15 & 23 & 14 & 17 & 31 & 21 & 25 & 28 & 18 & 20 \\
\hline XIX & 27 & 15 & 22 & 23 & 15 & 18 & 24 & 6 & 20 & 34 & 11 & 27 & 27 & 15 & 21 \\
\hline$X X I$ & 33 & 18 & 24 & 28 & 19 & 20 & 27 & 15 & 20 & 35 & 21 & 26 & 31 & 18 & 22 \\
\hline Outros & 35 & 19 & 27 & 28 & 18 & 19 & 28 & 14 & 20 & 36 & 21 & 30 & 32 & 19 & 23 \\
\hline Total & 30 & 18 & 23 & 26 & 17 & 19 & 25 & 13 & 20 & 35 & 21 & 27 & 29 & 18 & 21 \\
\hline
\end{tabular}

" $\mathrm{D}=$ Despesa-dia.

$\pm \mathrm{D}=$ Desvio-padrão da despesa-dia.

MED = Mediana da despesa-dia.

***: Capítulos CID-10 - Descrição: I - Algumas doenças infecciosas e parasitárias; II - Neoplasias (tumores); V - Transtornos mentais e comportamentais; VI - Doenças do sistema nervoso; VII - Doenças do olho e anexos; VIII - Doenças do ouvido e da apófise mastoide; IX - Doenças do aparelho circulatório; X - Doenças do aparelho respiratório; XI - Doenças do aparelho digestivo; XII - Doenças da pele e do tecido subcutâneo; XIII - Doenças sistema osteomuscular e tecido conjuntivo; XIV - Doenças do aparelho geniturinário; XIX - Lesões envenenamento e algumas outras consequências causas externas; XXI - Contatos com serviços de saúde.

\section{Discussão}

Essa pesquisa estima a prevalência, a duração e a despesa previdenciária dos ADAT e a influência de fatores como grupo de agravos segundo os capítulos da CID-10, o sexo e a idade do empregado segurado sobre esses indicadores para 2008.

Os grupos de agravos com as maiores prevalências de ADAT são o do capítulo das lesões (XIX), seguido pelo das doenças osteomusculares (XIII) e o das doenças mentais (V). A influência do sexo na prevalência de ADAT se mostra maior entre os homens, no entanto, quando excluído o capítulo das lesões (XIX), a prevalência do conjunto dos demais capítulos é superior entre as mulheres. Não há diferença na duração dos ADAT entre os sexos. A despesa média e a despesa-dia são maiores entre os homens e entre os empregados da faixa etária mais elevada. A prevalência estimada dos ADAT apresentada neste estudo é menor que a de benefícios por incapacidade decorrentes de acidentes do trabalho encontrada a partir de dados neozelandeses (NEW ZEALAND, 2008), canadenses (YASSI; GILBERT; CVITKOVICH, 2005) e franceses (FERRIE et al., 2009).

Essa menor prevalência de ADAT pode decorrer em parte dos diferentes critérios normativos para concessão dos benefícios, o que dificulta a comparação dos dados entre países. Enquanto no Brasil o benefício é concedido a partir do décimo sexto dia consecutivo da incapacidade para o trabalho, em outros países, como nos EUA, a depender do Estado, a con- cessão pode ocorrer a partir do terceiro ou do sétimo dia (USA, 2010). Acrescenta-se que, diferentemente do Brasil, há países que também consideram na mesma casuística os benefícios concedidos aos servidores públicos (NORDIN; BERGTSSON, 2001). Outra possível causa para a diferença entre as prevalências decorre diretamente do estágio no qual cada país se encontra em relação à diminuição da subnotificacação dos ATs. Nos países em desenvolvimento, essa questão ainda é um grande desafio a ser superado. Diversamente, nos países desenvolvidos, apesar de presente, a subnotificação é reconhecidamente menor (HÄMÄLÄINEN; TAKALA; SAARELA, 2006).

Apesar da maior prevalência de ADAT ser decorrente de lesões $(53,0 / 10.000)$, merecem destaque as doenças osteomusculares $(30,4 / 10.000)$ e as doenças mentais (3,7/10.000). Diferentemente das lesões, esses dois últimos grupos de doenças estão mais associados aos agravos crônicos do que aos agudos, o que dificulta o estabelecimento do nexo técnico entre o agravo e o trabalho. A partir de mudanças no processo de estabelecimento desse nexo pelo INSS, em abril de 2007, essa dificuldade vem sendo bastante diminuída, resultando em significativos aumentos na caracterização da incapacidade temporária para o trabalho como de natureza acidentária, principalmente em relação às doenças. Em 2006, foram concedidos 140.998 auxílios-doença por acidentes do trabalho, saltando para 274.946 em 2007 e para 356.336 em 2008 (BRASIL, 2009c). Vale ressaltar que esses aumentos não decorrem de excesso de notificação acidentária, mas sim de um processo de mi- 
gração dos benefícios que antes eram caracterizados como doenças comuns, apesar do forte componente ocupacional em doenças do trabalho (BARBOSA-BRANCO, 2008).

A presença das doenças osteomusculares e das mentais como segunda e terceira causas de ADAT, respectivamente, é coincidente com as estatísticas recentes apresentadas pelo Reino Unido, nas quais esses mesmos grupos de doenças foram identificados como as principais causas de incapacidade para o trabalho (UNITED KINGDOM, 2010). Nos EUA, as doenças osteomusculares também se destacam e representam 34,0\% das causas de afastamentos do trabalho (PEELE; XU; COLOMBI, 2005). Nos últimos anos, estudos têm mostrado o crescimento acentuado dos transtornos mentais relacionados ao trabalho (AUSTRALIAN SAFETY AND COMPENSATION COUNCIL, 2006; SELIGMANN-SILVA et al., 2010; VIRTANEN et al., 2008). Por outro lado, há países com baixas prevalências, provavelmente em decorrência das restrições legais para o estabelecimento de nexo entre o trabalho e o agravo. No Canadá, por exemplo, na maioria das províncias, o reconhecimento das doenças mentais relacionadas ao trabalho é restrito às decorrentes de eventos traumáticos (YASSI; GILBERT; CVITKOVICH, 2005).

A maior prevalência de ADAT em decorrência das lesões (XIX) entre os homens já havia sido observada por outras pesquisas (MEERDING, MULDER, BEECK, 2006; NEW ZEALAND, 2006; NORDIN; BERGTSSON, 2001). Uma das possíveis explicações para essa maior prevalência masculina deve-se ao fato dos homens estarem mais envolvidos em atividades de maior risco de acidentes, o que naturalmente leva a maiores ocorrências. Por outro lado, quando homens e mulheres estão expostos aos mesmos riscos, tem-se observado a maior prevalência de lesões no sexo feminino (OYEBODE et al., 2008). Entre as prováveis causas desta maior prevalência feminina, quando do desempenho das mesma atividades que os homens, estão as diferenças antropométricas entre os sexos, uma vez que as ferramentas, os equipamentos, os postos de trabalho foram desenvolvidos tendo como principal referência os homens. Outras possibilidades seriam que as mulheres recebem menos informações acerca da saúde e segurança no trabalho e que os homens têm mais autonomia e controle sobre o trabalho (GJESDAL; BRATBERG, 2002; OYEBODE, 2008).

A superioridade da prevalência des mulheres nos grupos de doenças de maior destaque (osteomusculares e mentais) vai ao encontro dos resultados apresentados por várias pesquisas (AUSTRALIAN SAFETY AND COMPENSATION COUNCIL, 2006; ISLAM et al., 2001; NYSTUEN; HAGEN; HERRIN, 2001; PRANSKY et al., 2006; NORDIN; BERGTSSON,
2001). Entre as potenciais explicações para o maior afastamento do trabalho entre as mulheres em decorrência das doenças do trabalho, encontram-se os problemas relativos à organização do trabalho, potencializados pelo acúmulo das atividades laborais com as atividades domésticas (HENSING; ANDERSSON; BRAGE, 2006), mas há também referências às flutuações hormonais, que levam à maior vulnerabilidade das mulheres aos fatores psicossociais, fisiológicos e ambientais (ANDRADE; VIANA; SILVEIRA, 2006). No entanto, um estudo finlandês mostra que, quando os resultados são controlados por ocupação e local de trabalho, a diferença das prevalências entre os sexos decresce significativamente (LAAKSONEN et al., 2010).

A maior prevalência de ADAT na faixa etária $\geq 40$ anos também foi constatada por diversas pesquisas, tanto no que se refere ao conjunto dos agravos (NEW ZEALAND, 2008; ROELEN et al., 2009), quanto em relação a grupos de doenças (AUSTRALIAN SAFETY AND COMPENSATION COUNCIL, 2006; NYSTUEN; HAGEN; HERRIN, 2001; PRANSKY, 2006). A excepcionalidade do capítulo das lesões (XIX) como o único a apresentar maior prevalência na menor faixa etária está em conformidade com outros resultados (MEERDING; MULDER; BEECK, 2006; OYEBODE, 2008). A idade avançada do trabalhador poderia ser considerada como potencial fator de proteção contra as lesões (XIX), provavelmente devido à maior experiência, à menor exigência física e à menor exposição aos riscos traumáticos quando comparados aos mais jovens.

Considerando o conjunto dos ADAT, a duração média $(75 \pm 64$ dias; mediana $=59$ dias $)$ neste estudo é inferior às apresentadas por outras pesquisas cujos resultados médios chegaram a 165 dias de incapacidade (CHEADLE et al., 1994; FULTON-KEHOE et al., 2000). Destacam-se as diferenças de duração média dos ADAT de acordo com os grupos de agravos dos capítulos CID-10. A flutuação de $54 \pm 33$ dias (mediana $=47$ dias) a $146 \pm 106$ dias (mediana $=121$ dias) está diretamente relacionada às características fisiológicas, patológicas e terapêuticas das mais diversas condições incapacitantes pelas quais os trabalhadores são acometidos, refletindo diretamente no período necessário para retorno ao trabalho. A maior duração dos ADAT decorrentes dos tumores, em 170,0\% em relação às doenças do aparelho digestivo, está associada à evolução natural de tratamento e retorno às atividades, independentemente de cura. Reconhece-se que a duração do tratamento, mesmo que parcial, de um tumor seja maior que o tratamento de uma infecção intestinal. As durações médias dos ADAT por doenças osteomusculares (80 \pm 70 dias; mediana $=62$ dias) e por doenças mentais ( $80 \pm 66$ dias; mediana $=65$ dias) encontradas 
são inferiores às encontradas em outros países para as lombalgias (HASHEMI et al., 1997; PRANSKY et al., 2006) e para as doenças mentais (AUSTRALIAN SAFETY AND COMPENSATION COUNCIL, 2006).

A semelhança da duração média entre os sexos encontrada para o conjunto dos ADAT não se mantém quando esse indicador é analisado segundo o capítulo CID-10. Neste estudo, a maior duração dos benefícios entre o sexo masculino decorrentes de lesões (XIX), de doenças osteomusculares (XIII) e doenças respiratórias (X) é contrária aos resultados apresentados por vários pesquisadores (OYEBODE, 2008; PEELE; XU; COLOMBI, 2005; PRANSKY et al., 2006). Por outro lado, a maior duração média encontrada entre o sexo masculino para os ADAT decorrentes de doenças mentais é corroborada por estudo norueguês (NYSTUEN; HAGEN; HERRIN, 2001).

A relação direta entre a duração da incapacidade e a idade do empregado também foi apresentada por outra pesquisa (CHEADLE et al., 1994). É razoável que os empregados nas maiores faixas etárias necessitem de maior tempo para retorno às atividades quando acometidos por agravos da mesma natureza. Várias pesquisas apontam maior duração da incapacidade para o trabalho decorrente dos transtornos musculoesqueléticos (HAGEN; THUNET, 1998; PEELE; XU; COLOMBI, 2005; PRANSKY et al., 2006) e das doenças mentais (NYSTUEN; HAGEN; HERRIN, 2001) entre os trabalhadores nas faixas etárias mais elevadas. Várias têm sido as explicações para esse quadro, destacando-se a maior gravidade e consequente necessidade de maior tempo para recuperação (PEELE; XU; COLOMBI, 2005; PRANSKY et al., 2006). Cheadle e colaboradores (1994) apontam como potencial causa de maior duração entre os de maior idade, a dificuldade de reinserção no mercado de trabalho. No entanto, no caso específico dos ADAT entre os empregados brasileiros, essa não seria uma preocupação imediata, uma vez que lhes é assegurada a estabilidade no emprego por doze meses após o retorno ao trabalho (BRASIL, 1991).

A despesa média é diretamente influenciada por dois fatores, pela duração da incapacidade e pelo valor do salário-benefício (BRASIL, 1991). Considerando os ADAT por agravos, segundo os capítulos da CID-10, a maior despesa média é relativa ao capítulo das neoplasias, sendo preponderantemente influenciada pela duração, na qual a média está 194,7\% acima da média dos capítulos em conjunto. Apesar de ter sido em menor grau, a média do salário-benefício dos trabalhadores afastados por neoplasias também exerceu influência sobre a despesa média, uma vez que está 11,9\% acima da média dos trabalhadores afastados por todos os agravos conjuntamente.
A despesa-dia é essencialmente influenciada pelo salário-benefício. A maior despesa-dia é no capítulo das doenças mentais (V), no qual o salário-benefício médio dos empregados é o maior entre os demais. A menor despesa-dia é no capítulo das doenças da pele e do tecido subcutâneo (XII), no qual o salário-benefício médio é o menor entre os demais. Estariam os trabalhadores melhor remunerados mais sujeitos às doenças mentais do que às doenças da pele e do tecido subcutâneo? Aponta-se uma possível associação entre remuneração e causa de afastamento que necessita ser estudada. Pesquisa canadense também resultou em maiores despesas de benefícios relativos aos afastamentos temporários do trabalho em decorrência das doenças mentais (DEWA; CHAU; DERMER, 2010). Publicação americana de 1994 já ressaltava a necessidade de se dar importância ao impacto econômico das doenças mentais, entre elas, principalmente as desordens depressivas. A pesquisa destacou a maior duração da incapacidade decorrente dos transtornos mentais em relação a outras condições incapacitantes, tais como doenças do coração, hipertensão arterial, dorsalgias, diabetes e outras (CONTI; BURTON, 1994).

Uma vez que não houve diferença de duração média dos ADAT entre os sexos, os valores a maior da despesa média e da despesa-dia dos benefícios entre os empregados masculinos em relação aos femininos decorrem do maior salário-benefício médio entre os homens em detrimento das mulheres. Em 2008, essa diferença foi de 17,6\%. Pesquisa associa menor despesa entre as mulheres à predominância das mulheres em ocupações que historicamente pagam menores salários (ISLAM et al., 2001). A superioridade da despesa média dos trabalhadores com idade $\geq 40$ anos em relação aos demais decorre da maior duração média dos ADAT, bem como do maior salário-benefício médio nessa faixa etária. A despesa-dia decorre exclusivamente do maior salário-benefício. Em 2008, o salário-benefício médio dos empregados com idade $\geq 40$ anos foi $17,1 \%$ superior ao dos empregados com idade $<40$ anos. Os dados relativos aos salários-benefício dos empregados não foram apresentados nos resultados. Pesquisa aponta maior despesa de reposição salarial em decorrência das doenças osteomusculares nas faixas etárias mais elevadas (PEELE; XU; COLOMBI, 2005).

A maior limitação desta pesquisa está relacionada à população de estudo, que não compreende a totalidade da população trabalhadora do Brasil na medida em que exclui, entre os segurados do RGPS, os contribuintes individuais, os empregados domésticos, os segurados especiais e os facultativos, além dos servidores públicos e dos trabalhadores informais. É possível que os fatores de risco aos quais esses trabalhadores estejam expostos sejam tão ou 
mais importantes para a saúde do que os contemplados neste estudo. Apesar dessa limitação, destaca-se a representatividade da população estudada, a qual corresponde a 43,8\% da população ocupada (INSTITUTO BRASILEIRO DE GEOGRAFIA E ESTATÍSTICA, 2010) e a 80,6\% dos segurados do RGPS. Apesar de não ter sido analisada a incapacidade para o trabalho desses grupos de trabalhadores, eles compartilham com bastante frequência dos mesmos fatores de risco aos quais os trabalhadores da população de estudo estão expostos.

Acrescenta-se que o SUB seja uma das maiores bases de dados previdenciárias do mundo sob uma única administração. Esse fator traz vantagens para a homogeneidade das informações, uma vez que a concessão dos benefícios segue procedimentos e protocolos padronizados. Ressalta-se ainda que, em decorrência de importantes modificações ocorridas nos últimos anos relativas à implementação do NTEP (BRASIL, 2007), a base de dados utilizada passou por substanciais melhorias visando ampliar a consistência e a confiabilidade das informações.

\section{Conclusões}

Em 2008, o Brasil apresentou baixas taxas de prevalência dos benefícios por incapacidade temporária para o trabalho de natureza acidentária quando comparados com países desenvolvidos. É possível que o panorama traçado neste estudo decorra, em grande parte, de mecanismos de subnotificação por parte dos empregadores, tais como a não emissão da CAT, como também possam decorrer de diferentes critérios normativos para concessão do benefício entre os países comparados.

Apesar dos avanços ocorridos a partir de 2007, há muito a percorrer, principalmente em relação ao estabelecimento do nexo entre o trabalho e o agravo para as doenças, sobretudo para aquelas nas quais o período de latência é longo.

No Brasil, as lesões (XIX) ainda representam a maioria dos casos dos ADAT, o que aponta para a persistência da precariedade das medidas de segurança nos ambientes do trabalho. Diferentemente do Brasil, em países desenvolvidos as doenças do trabalho representam a maioria dos casos de incapacidade de natureza acidentária (PEELE; XU; COLOMBI, 2005; UNITED KINGDOM, 2010). É extremamente difícil vislumbrar que em um futuro próximo o Brasil consiga prevenir de forma efetiva as doenças do trabalho, uma vez que, até o momento, não tenha conseguido superar as elevadas taxas de acidentes de origem traumática.

Para a diminuição das despesas, os dados apresentados permitem concluir que, além das medidas preventivas, uma vez ocorrido o agravo, faz-se necessário reduzir ao mínimo a duração da incapacidade. Essa minimização pode ser feita por meio de diagnóstico e tratamento precoces, bem como por melhoria do processo de reabilitação profissional. É importante nesse processo contar com o compromisso legal do empregador em adaptar o ambiente e o processo de trabalho para inserir o trabalhador em uma atividade compatível com suas condições laborais.

\section{Agradecimentos}

Os autores agradecem ao Dr. Paulo Rogério Albuquerque de Oliveira, Coordenador-Geral de Políticas de Seguro Contra Acidentes do Trabalho e Relacionamento Interinstitucional do Ministério da Previdência Social (MPS), pela importante colaboração na obtenção dos dados.

\section{Contribuições de autoria}

Ambos os autores foram responsáveis pela elaboração do projeto, pela revisão bibliográfica, pelo levantamento e pela análise dos dados e aprovaram a versão final do manuscrito.

\section{Referências}

ANDRADE, L. H. S. G.; VIANA, M. C.; SILVEIRA, C. M. Epidemiologia dos transtornos psiquiátricos nas mulheres. Revista de Psiquiatria Clínica, São Paulo, v. 33, n. 2, p. 43-54, 2006.

AUSTRALIAN SAFETY AND COMPENSATION COUNCIL. Work-related mental disorders in Australia. Australia: Commonwealth of Australia, 2006.
BARBOSA-BRANCO, A. O impacto do NTEP na caracterização dos acidentes de trabalho no Brasil. Revista CIPA - Caderno Informativo de Prevenção de Acidentes, São Paulo v. 29, n. 345, p. 50-55, 2008.

BARBOSA-BRANCO, A.; ROMARIZ, M. S. Doenças das cordas vocais e sua relação com o trabalho. Comunicação em Ciências da Saúde, Brasília, v. 17, n. 1, p. 37-45, 2006. 
BENAVIDES, G. B. et al. Occupational categories and sickness absence certified as attributable to common diseases. European Journal of Public Health, Netherlands, v. 14, n. 1, p. 51-55, 2003.

BINDER, M. C. P.; CORDEIRO, R. Sub-registro de acidentes do trabalho em localidade do Estado de São Paulo. Revista de Saúde Pública, São Paulo, v. 37, n. 4, p. 409-416, 1997.

BRASIL. Instrução Normativa INSS/PRES nº 16, de 27 de março de 2007. Dispõe sobre procedimentos e rotinas referentes ao Nexo Técnico Epidemiológico Previdenciário - NTEP, e dá outras providências. Diário Oficial [da] República Federativa do Brasil, Brasília, DF, 30 mar. 2007. Disponível em: < http://www010. dataprev.gov.br/sislex/paginas/38/INSS-PRES/2007/16. htm>. Acesso em: 01 dez. 2011.

. Lei $\mathrm{n}^{\circ}$ 4.320, de 17 de março de 1964. Estatui normas gerais de direito financeiro para elaboração e controle dos orçamentos e balanços da União, dos Estados, dos Municípios e do Distrito Federal. Diário Oficial [da] República Federativa do Brasil, Brasília, DF, 23 mar 1964. Disponível em: < http://www010.dataprev. gov.br/sislex/paginas/42/1964/4320.htm>. Acesso em: 01 dez. 2011.

. Lei no ${ }^{\circ}$ 8.213, de 24 de julho de 1991. Dispõe sobre os planos de benefícios da Previdência Social e dá outras providências. Diário Oficial [da] República Federativa do Brasil, Brasília, DF, 14 ago. 1991. Disponível em: <http://www010.dataprev.gov.br/sislex/ paginas/42/1991/8213.HTM>. Acesso em: 04 dez. 2011.

. Ministério da Previdência Social. Seção IV - Acidentes do trabalho. Anuário Estatístico da Previdência Social - AEPS 2008, Brasília, DF, v. 17, p. 497, 2009a. Disponível em: <http://www.mps.gov.br/ arquivos/office/3_091028-191015-957-545.pdf > . Acesso em: 17 dez. 2010.

Ministério da Previdência Social. Seção V - Contribuintes da Previdência Social: capítulo 32 - Contribuintes empregados. Anuário Estatístico da Previdência Social - AEPS 2008, Brasília, DF, v. 17, p. 566-582, 2009b. Disponível em: <http://www.mps.gov. br/arquivos/office/3_091028-191015-957.pdf>. Acesso em: 17 dez. 2010.

. Ministério da Previdência Social. Seção I-Benefícios concedidos. Anuário Estatístico da Previdência Social - AEPS 2008, Brasília, DF, v. 17, p. 19, 2009c. Disponível em: < http://www.mps.gov.br/ arquivos/office/3_091028-191015-957.pdf>.Acesso em: 17 dez. 2010.

. Ministério da Previdência Social. Grandes números da Previdência Social. Boletim Estatístico da Previdência Social, v. 15, n. 5, p. 2, 2010. Disponível em: <http://www.mps.gov.br/arquivos/ office/3 100623-151859-804.pdf > . Acesso em: 04 nov. 2010.

. Ministério da Saúde. Departamento de Informática do SUS. Classificação estatística internacional de doenças e problemas relacionados à saúde - CID-10. 10. ed. rev. 2011. Disponível em <http://www.datasus.gov.br/cid10/v2008/cid10.htm>. Acesso em: 21 nov. 2011.
CHEADLE, A. et al. Factors influencing the duration of work-related disability: a population-based study of Washington State workers' compensation. American Journal of Public Health, Washington, DC, v. 84, n. 2, p. 190-196, 1994.

CONTI, D. J.; BURTON, W. N. The economic impacts of depression in a workplace. Journal of Occupational Medicine, Chicago, v. 36, n. 9, p. 983-988, 1994.

DEWA, C. S.; CHAU, N.; DERMER, S. Examining the comparative incidence and costs of physical and mental health-related. Journal of Occupational and Environmental Medicine, London, v. 52, n. 7, p. 758762, 2010

EUROPE. European Forum of the Insurance Against Accidents at Work and Occupational Diseases. Costs and funding of occupational diseases in Europe, 2004. Disponível em: < http://www.europeanforum.org/pdf/ Eurogip-08_E-cost.pdf>. Acesso em: 05 jul. 2010.

FACCHINI, L. A. et al. Sistema de informação em saúde do trabalhador: desafios e perspectivas para o SUS. Ciência e Saúde Coletiva, Rio de Janeiro, v. 10, n. 4, p. 857-867, 2005.

FERRIE, J. E. et al. Diagnose-specific sickness absence and all cause mortality in the GAZEL study. Journal of Epidemiology and Community Health, v. 63, n. 1, p. 50-55, 2009.

FREEMANA, K. et al. An actuarial method for estimating the long-term, incidence-based costs of Navy civilian occupational injuries and illnesses. Journal of Safety Research, United States of America, v. 32, n. 3, p. 289-291, 2001.

FULTON-KEHOE, D. et al. Years of productivity lost among injured workers in Washington State: modeling disability burden in workers' compensation. American Journal of Industrial Medicine, United States of America, v. 37, n. 6, p. 656-662, 2000.

GJESDAL, S.; BRATBERG, E. The role of gender in long-term sickness absence and transition to permanent disability benefits. Results from a multiregister based, prospective study in Norway 1990-1995. European Journal of Public Health, Netherlands, v. 12, p. 180-186, 2002.

HAGEN, B. K.; THUNET, O. Work incapacity from low back pain in the general population. Spine Jounal, United States of America, v. 23, n. 19, p. 2091-2095, 1998.

HÄMÄLÄINEN, P.; TAKALA, J.; SAARELA, K. L. Global estimates of occupational accidents. Safety Science, v. 44, p. 137-156, 2006.

HASHEMI, L. et al. Length of disability and cost of workers' compensation low back pain claims. Journal of Occupational Environmental Medicine, London, v. 39, n. 10, p. 937-945, 1997.

HENSING, G.; ANDERSSON, L. BRAGE, S. Increase in sickness absence with psychiatric diagnosis in Norway: a general population-based epidemiologic study of age, gender and regional distribution. BMC Medicine, London, v. 4, n. 19, 22, Aug. 2006. 
INSTITUTO BRASILEIRO DE GEOGRAFIA E ESTATÍSTICA. Conceitos população em idade ativa. População economicamente ativa. População ocupada, Rio de Janeiro: IBGE, 2010. Disponível em: < http:// www.ibge.gov.br/home/estatistica/indicadores/ trabalhoerendimento/pme/pmemet2.shtm > . Acesso em: 03 jan. 2011.

INTERNATIONAL LABOUR ORGANIZATION. Work hazards kill millions, cost billions. Safety in numbers: pointers for the global safety at work, Switzerland: ILO, 23 maio 2003. Disponível em: <http://www.ilo.org/ global/About_the_ILO/Media_and_public_information/ Feature_stories/lang--en/WCM̄S_075615/iñdex.htm > . Acesso em: 09 mai 2010.

ISLAM, S. S. et al. Gender differences in work-related injury/illness: analysis of workers compensation claims. American Journal of Industrial Medicine, United States of America, v. 39, n. 1, p. 84-91, 2001.

LAAKSONEN, M. et al. Gender differences in sickness absence - the contribution of occupational and workplace. Scandinavian Journal of Work Environment and Health, Helsinki, v. 36, n. 5, p. 394-403, 2010.

LARSSON, T. J.; BETTS, N. J. The variation of occupational injury cost in Australia: estimates based on a small empirical study. Safety Science, v. 24, n. 2, p.143-155, 1996.

LEIGH, J. P.; McCURDY, S. A. Differences in workers' compensation disability and impairment ratings under old and new California law. Journal of Occupational and Environmental Medicine, London, v. 48, n. 4, p. 419-425, 2006.

LUNES, R. F. III - Impacto econômico das causas externas no Brasil: um esforço de mensuração. Revista de Saúde Pública, São Paulo, v. 31, n. 4, p. 38-46, 1997.

MEERDING, W. J.; MULDER, S.; BEECK, A. F. Incidence and costs of injuries in the Netherlands. European Journal of Public Health, London, v. 16, n. 3, p. 271-277, 2006.

MORAES, E. et al. Conceitos introdutórios de economia da saúde e o impacto social do abuso de álcool. Revista Brasileira de Psiquiatria, São Paulo, v. 28, n. 4, p. 321325, 2006.

NEW ZEALEND. Department of Labor. Work-related injury Claims - 2007. Wellington: DOL, 5 nov. 2008. Disponível em: <http://www.dol.govt.nz/publications/ lmr/lmr-injury-summary.asp >. Acesso em: 24 ago. 2010.

NORDIN, H.; BERGTSSON, B. Occupational accidents and work-related diseases in Sweden. Swedish: Swedish Work Environment Authority. Central Supervision Department. Statistics Division, 2001. Disponível em: <http://www.av.se/dokument/ inenglish/statistics/work-related_diseases2004.pdf $>$. Acesso em: 10 out. 2010.

NYSTUEN. P.; HAGEN, K. B.; HERRIN J. Mental health problems as a cause of long-term sick leave in the Norwegian workforce. Scandinavian Journal of Public Health, v. 29, n. 3, p. 175-182, 2001.

OLIVEIRA-ALBUQUERQUE, P. R.; BARBOSA-BRANCO, A. Nexo técnico epidemiológico previdenciário NTEP, fator acidentário de prevenção FAP: um novo olhar sobre a saúde do trabalhador. São Paulo: LTR, 2008.

OYEBODE, A. et al. Sex differences in injury patterns among workers in heavy manufacturing. American Journal of Epidemiology, Baltimore, v. 169, n. 2, p. 161166, 2008.

PEELE, P.B.; XU, Y.; COLOMBI, A. Medical care and lost work days costs in musculoskeletal disorders: older versus younger workers. International Congress Series, v. 1280, p. 214-218, 2005.

PRANSKY, G. S. et al. Length of disability prognosis in acute occupational low back pain claims. Spine Jounal, United States of America, v. 31, n. 6, p. 690-697, 2006.

RIKHARDSSON, P. M. Accounting for the cost of occupational accidents. Corporate Social Responsibility and Environmental Management, v. 11, n. 2, p. 63-70, 2004.

ROELEN, C. A. et al. Trends in the incidence of sickness absence due to common mental disorders between 2001 and 2007 in the Netherlands. European Journal of Public Health, London, v. 19, n. 6, p. 625-630, 2009.

ROSENMAN, K. D. et al. How much work-related injury and illness is missed by the current National Surveillance System? Journal of Occupational and Environmental Medicine, London, v. 48, n. 4, p. 357365, 2006.

SALINAS-TOVAR, J. S. et al. El subregistro potencial de accidentes de trabajo en el Instituto Mexicano del Seguro Social. Revista de Salud Pública de México, México, v. 46, n. 3, p. 204-209. 2004.

SANTANA, V.; NOBRE, L.; WALDVOGEL, B. C. Acidentes de trabalho no Brasil entre 1994 e 2004: uma revisão. Ciência e Saúde Coletiva, Rio de Janeiro, v. 10, n. 4, p. 841-855, 2005.

SANTANA, V. S. Bases epidemiológicas do fator acidentário previdenciário. Revista Brasileira de Epidemiologia, Rio de Janeiro, v. 8, n. 4, p. 440-453, 2005.

SELIGMANN-SILVA, E. et al. O mundo contemporâneo do trabalho e a saúde mental do trabalhador. Revista Brasileira de Saúde Ocupacional, São Paulo, v. 35, n. 122, p. 187-191, 2010.

UNITED KINGDOM. Health and Safety Executive. The annual statistics report 2009/10. United Kingdom: HSE, 2010. Disponível em: <http://www.hse.gov.uk/ statistics/>. Acesso em: 03 jan. 2011.

VIRTANEN, M. et al. Contribution of non-work and work-related risk factors to the association between income and mental disorders in a working population: the health 2000 study. Occupational and Environmental Medicine, London, v. 65, n. 3, p. 171178, 2008.

YASSI, A.; GILBERT, M.; CVITKOVICH, Y. Trends in injuries, illnesses, and policies in Canadian healthcare workplaces. Canadian Journal of Public Health, Ottawa, v. 96, n. 5, p. 333-339, 2005. 